\title{
Análise da incidência temporal, espacial e de tendência de fogo nos biomas e unidades de conservação do Brasil
}

\author{
Analysis of the temporal, spatial and trend incidence of fire in biomes and \\ conservation units of Brazil
}

\author{
Janisson Batista de Jesus ${ }^{\mathrm{I}}$, Cristiano Niederauer da Rosa ${ }^{\mathrm{II}}$, \\ Íkaro Daniel de Carvalho Barreto ${ }^{\mathrm{III}}$, Milton Marques Fernandes ${ }^{\mathrm{IV}}$
}

\begin{abstract}
Resumo
O Brasil tem extensas formações vegetais ao longo do seu território que são afetadas por ocorrências de queimadas, necessitando de um monitoramento espaço-temporal e de estudos que relacionem a sua atuação nos biomas e nas áreas protegidas do país. Sendo assim, o objetivo do presente estudo foi analisar o comportamento temporal e espacial das ocorrências de fogo de 2003 a 2017 nos biomas brasileiros: Amazônia, Caatinga, Cerrado, Mata Atlântica, Pantanal e Pampa, incluindo as incidências nas áreas de Unidades de Conservação, verificando possíveis padrões da distribuição dos focos, assim como a tendência estatística das suas ocorrências. Foram utilizados dados geoespaciais de 2003 a 2017 do satélite de referência AQUA_M-T obtido no Instituto Nacional de Pesquisas Espaciais, e das Unidades de Conservação disponibilizados pelo Ministério do Meio Ambiente. Estatisticamente, foi aplicado o algoritmo Kernel para analisar a distribuição espacial a partir da média da série temporal estudada, e o teste não paramétrico de Mann-Kendall, considerando também a sua Persistência de Longo Alcance para verificar a tendência de ocorrência. Observou-se que os biomas Amazônia e Cerrado possuem, respectivamente, os maiores focos de fogo, com o Cerrado tendo maior área queimada. Consequentemente, as Unidades de Conservação localizadas nestes biomas tiveram maior quantidade de focos detectados. A análise da tendência estatística indicou que o crescimento da ocorrência de fogo possui sazonalidade em todos os biomas, com o Pampa sendo caracterizado por uma condição natural. Portanto, o estudo permitiu identificar e quantificar, espacialmente, os maiores focos de queimadas nos Biomas brasileiros, bem como nas Unidades de Conservação em cada respectivo bioma para a série estudada, servindo como base para medidas de prevenção e controle do fogo principalmente nas Unidades de Conservação que possuem maiores ocorrências no país.
\end{abstract}

Palavras-chave: Monitoramento ambiental; Áreas protegidas; Geoprocessamento; Teste de Mann-Kendall

Engenheiro Florestal, Doutorando em Sensoriamento Remoto, Programa de Pós-Graduação em Sensoriamento Remoto, Universidade Federal do Rio Grande do Sul, Av. Bento Gonçalves, 9500, CEP 91501-970, Porto Alegre (RS), Brasil. janisson.eng@gmail.com (ORCID: 0000-0001-8372-5557)

II Tecnólogo em Geoprocessamento, Doutorando em Sensoriamento Remoto, Programa de Pós-Graduação em Sensoriamento Remoto, Universidade Federal do Rio Grande do Sul, Av. Bento Gonçalves, 9500, CEP 91501-970, Porto Alegre (RS), Brasil. cristianonrd@gmail.com (ORCID: 0000-00033693-4764)

III Estatístico, Doutorando em Biometria e Estatística Aplicada, Programa de Pós-Graduação em Biometria e Estatística Aplicada, Universidade Federal Rural de Pernambuco, Rua Dom Manoel de Medeiros, s/n, CEP 52171-900, Recife (PE), Brasil. daniel.carvalho.ib@gmail.com (ORCID: 00000001-7253-806X)

IV Engenheiro Florestal, Dr., Professor do Departamento de Ciências Florestais, Universidade Federal de Sergipe, Av. Marechal Rondon, s/n, CEP 49100-000, São Cristóvão (SE), Brasil. miltonmf@gmail.com (ORCID: 0000-0002-9394-0020) 


\begin{abstract}
Brazil has extensive plant formations throughout its territory that are affected by burning occurrences, necessitating space-time monitoring and studies that relate its performance to the biomes and protected areas of the country. Therefore, the objective of the present study was to analyze the temporal and spatial behavior of the occurrences of fires from 2003 to 2017 in the Brazilian biomes: Amazonia, Caatinga, Cerrado, Mata Atlântica, Pantanal and Pampa, including incidences in the areas of Conservation Units, possible patterns of distribution of forest fire outbreaks, as well as the statistical trend of its occurrence. Geospatial data were used from 2003 to 2017 of the AQUA_M-T reference satellite obtained from the National Space Research Institute and from the Conservation Units provided by the Ministry of the Environment. Statistically, the Kernel algorithm was applied to analyze the spatial distribution from the mean of the time series studied, and the non-parametric Mann-Kendall test, also considering its Long Range Persistence to verify the tendency of the occurrence. It was observed that the Amazon and Cerrado biomes have, respectively, the largest fires, with the Cerrado having a larger area burned. Consequently, the Conservation Units located in these biomes had a higher amount of fires. The tendency statistical analysis indicated that the growth of the occurrence of fire has seasonality in all the biomes, with the Pampa being characterized by a natural condition. Therefore, the study allowed to quantify and to identify, spatially, the largest fires in the Brazilian Biomes, as well as in the Conservation Units in each respective biome for the studied series, serving as a basis for fire prevention and control measures, mainly in which that have the highest occurrences in the country.
\end{abstract}

Keywords: Environmental monitoring; Protected areas; Geo-processing; Mann-Kendall test

\title{
Introdução
}

Os incêndios florestais têm uma vasta incidência ao longo dos variados biomas, das Unidades de Conservação (UC), das áreas de preservação, das áreas de reflorestamento, das fazendas, ou até mesmo margens de estradas e proximidades de aglomerados urbanos, ocasionando, em muitos casos, diversos prejuízos ecológicos, paisagísticos e econômicos (COSTA et al., 2009), tornando-se imprescindível o conhecimento e acompanhamento destes eventos.

O monitoramento e entendimento das ocorrências de focos de queimadas são essenciais para compreender a sua interação com os processos ecológicos e os impactos provenientes das atividades humanas em múltiplas escalas espaciais de análise, gerando informações que podem subsidiar alternativas estratégicas para a conservação da biodiversidade e do solo, e a redução das emissões de carbono (ALVEZ; PÉREZ-CABELLO, 2017). Além disto, é relevante destacar a relação clima-fogo neste processo, identificando as áreas mais sensíveis às queimadas, provocadas pelo tipo de material combustível, estando associado diretamente ao tipo de floresta uma vez que os ecossistemas podem ser arranjados em um gradiente de nível aridez ao longo do qual o fogo é restringido pela disponibilidade de combustível (incêndios movidos pelo material disponível), e pelo potencial da ocorrência da queima causado pela seca (BEDIA et al., 2015).

O planejamento e a implementação do gerenciamento dos incêndios florestais, a predição do risco e prevenção de suas ocorrências podem ser baseadas nos mapeamentos espaciais e temporais decorrentes de técnicas de sensoriamento remoto, tendo alto potencial no monitoramento da vegetação afetada pelo fogo (FRANKE et al., 2018; SANTOS et al., 2018). 
Para isto, é necessário identificar os locais de maiores ocorrências de incêndios florestais, quantificando-os e observando as suas mudanças ao longo do tempo e em termos de escala, ou seja, nos padrões temporais e espaciais das queimadas e seus atributos (como: intensidade, sazonalidade, tamanho e tempo de retorno) para entender a dinâmica do fogo (ALVARADO et al., 2017).

O conhecimento da distribuição espacial do fogo, como a sua extensão, possibilita a elaboração de mapas temáticos que permitem localizar regiões com maiores e menores riscos de ocorrências de incêndios de maiores dimensões ao longo das diferentes paisagens e tipos de ecossistemas florestais, podendo fornecer informações importantes para o planejamento das ações de prevenção e combate, minimizando os efeitos negativos causados pelo fogo por meio da gestão dos elementos que compõem a paisagem, priorizando técnicas que reduzam o perigo de incêndio em nível de mancha, diminuam a conectividade entre coberturas inflamáveis, e aumentem a heterogeneidade da paisagem (MAGALHÃES et al., 2017).

No Brasil, a detecção e o monitoramento operacional dos focos de queimadas e de incêndios florestais detectados orbitalmente por satélites, o cálculo e a previsão do risco de fogo da vegetação são realizados através do Programa Queimadas sob desenvolvimento e responsabilidade do Instituto Nacional de Pesquisas Espaciais (INPE). Vários estudos já abordam o tema sobre os incêndios florestais, entretanto, existem poucas pesquisas que abordam a espacialização ao longo dos biomas brasileiros, sendo verificado apenas um estudo nesse viés (ARAÚJO; FERREIRA; ARANTES, 2012), sendo que os demais estudos somente avaliam biomas ou locais específicos como em Unidades de Conservação conforme visto em Torres et al. (2016) e Lima et al. (2018), áreas estas que são protegidas pela Lei 9.985/2000 através do Sistema Nacional de Unidades de Conservação (MMA, 2011), e que, por sua vez, assumem relevante importância para salvaguardar, entre outras características, os ecossistemas de uma determinada região e merecem atenção e preocupação quanto à ocorrência das queimadas.

Portanto, devido à escassez de estudos de queimadas considerando as várias formações vegetacionais e as áreas protegidas do Brasil, o objetivo do presente estudo foi analisar as ocorrências de fogo de 2003 a 2017 nos biomas brasileiros: Amazônia, Caatinga, Cerrado, Mata Atlântica, Pantanal e Pampa, avaliando o seu comportamento espaço-temporal, incluindo as incidências nas áreas de Unidades de Conservação, a fim de identificar possíveis padrões da distribuição dos focos de incêndio florestal/queimadas nestes tipos de vegetação. Além disto, também foi analisada a tendência estatística da sua ocorrência nas fitofisionomias analisadas.

\section{Material e métodos}

\section{Área de estudo}

O estudo foi realizado em todo o território brasileiro, que tem $8.516 .000 \mathrm{~km}^{2}$, analisando os seis biomas brasileiros: Amazônia, Caatinga, Cerrado, Mata Atlântica, Pampa e Pantanal, os quais têm sua distribuição espacial representada na Figura 1, cada um com a sua abrangência ao longo do território, suas fisionomias específicas e características próprias. 


\section{Figura 1 - Distribuição espacial dos biomas brasileiros: Amazônia, Caatinga, Cerrado, Mata Atlântica, Pampa e Pantanal nas regiões do Brasil}

Figure 1 - Spatial distribution of Brazilian biomes: Amazonia, Caatinga, Cerrado, Atlantic Forest, Pampa and Pantanal in the Brazilian regions

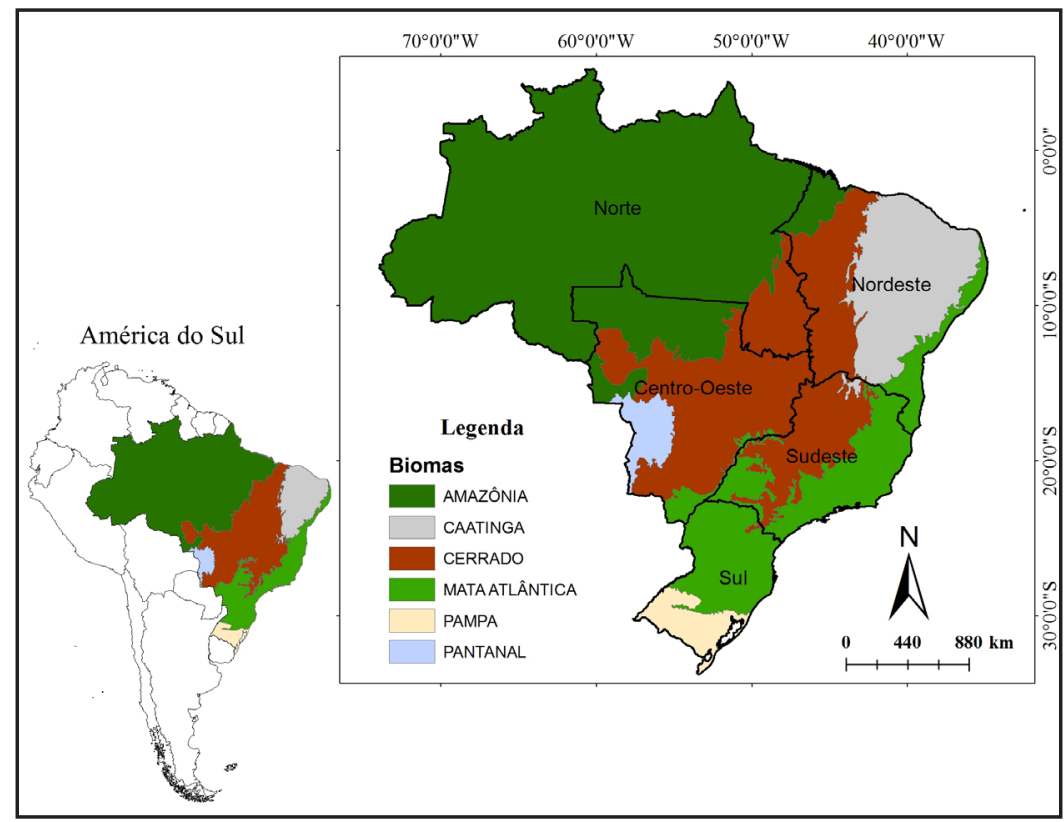

Fonte: Jesus et al. (2019)

O bioma Amazônico é o maior do Brasil, ocupando cerca de 49,29\% do território nacional com 4.196.943 km² (IBGE, 2004), com grande expansão na região Norte do país. Típica floresta de porte alto, e que estimativas a indicam como a maior reserva de madeira tropical do mundo. Além disso, a bacia hidrográfica amazônica também se destaca, tendo como rio principal o Amazonas, por ser a maior do mundo, tendo 1.100 afluentes e cobrindo cerca de 6 milhões de $\mathrm{km}^{2}$ e tem 1.100 afluentes (MMA, 2018a).

A Caatinga ocupa cerca de $11 \%$ do território nacional, ocupando umaárea de aproximadamente $844.453 \mathrm{~km}^{2}$, englobando parte significativa da região Nordeste e uma pequena parte ao norte do Sudeste (MMA, 2018). A sua vegetação é associada a ambientes com baixa disponibilidade hídrica, e, portanto, com significativo grau de aridez durante determinado período de tempo, classificada como: Floresta Subcaducifólia, Floresta Caducifólia, Caatinga Hipoxerófila e Caatinga Hiperxerófila (BRASIL, 1972).

O Cerrado ocupa uma área de $2.036 .448 \mathrm{~km}^{2}$, cerca de $22 \%$ do território nacional, sendo o segundo maior bioma da América do Sul (MMA, 2018), sendo observado nas regiões Norte, Nordeste, Sudeste e Centro-Oeste. É formado pelas fisionomias de: Campo Limpo, Campo Sujo, Campo Rupestre, Cerrado, Cerradão, Matas Secas, ciliares e galeria, e Veredas (EMBRAPA, 2018).

A Mata Atlântica está distribuída ao longo de quase toda a faixa continental Atlântica leste do país e ocupava, originalmente, mais de 1,3 milhões de $\mathrm{km}^{2}$ em 17 estados do território brasileiro, porém, atualmente, restam cerca de $29 \%$ de sua cobertura original. É composta por: Floresta Ombrófila Densa; Floresta Ombrófila Mista; Floresta Ombrófila Aberta; Floresta Estacional Semidecidual; e Floresta Estacional Decidual, e ecossistemas associados (manguezais, vegetações de restingas, campos de altitude, brejos interioranos e encraves florestais do Nordeste) (MMA, 2018a).

O Pampa é um bioma de campos típico da zona temperada da América do Sul, estando na região Sul do Brasil e restrita ao estado do Rio Grande do Sul, ocupando $178.243 \mathrm{~km}^{2}$, correspondendo a 2,07\% do território nacional (MMA, 2018a). 
O Pantanal é considerado uma das maiores extensões úmidas contínuas do planeta, apesar de ser o de menor extensão no Brasil, ocupando 1,76\% da área total do território. Este bioma sofre influência direta de três importantes biomas brasileiros: Amazônia, Cerrado e Mata Atlântica, e por ser uma planície aluvial também é influenciado por rios que drenam a bacia do Alto Paraguai, e pelo bioma chaco (denominação dada ao Pantanal localizado no norte do Paraguai e leste da Bolívia) (MMA, 2018a).

\section{Obtenção e análise dos dados}

Os dados dos focos de fogo no Brasil foram obtidos no banco de dados do Programa Queimadas desenvolvido pelo Instituto Nacional de Pesquisas Espaciais (INPE, 2018), utilizando os arquivos tabulados e shapefile dos anos de 2003 a 2017, num total de 15 anos, a respeito de todas as queimadas ou incêndios florestais identificadas em todo o território brasileiro analisando-as anualmente e mensalmente, este obtendo a média de cada mês da série histórica. Foi utilizado o satélite de referência AQUA_M-T, o qual, conforme o INPE (2018), é indicado para as análises de séries temporais de eventos de queimadas, analisando especificamente o número de focos e área queimada de cada bioma, bem como as ocorrências observadas nas delimitações das Unidades de Conservação.

Os dados vetoriais das Unidades de Conservação disponibilizados pelo Cadastro Nacional de Unidades de Conservação (CNUC) foram obtidos diretamente no endereço Ministério do Meio Ambiente (MMA, 2018b), os quais estão divididos em seis grupos: UCs Federais de Proteção Integral e de Uso Sustentável, UCs Estaduais de Proteção Integral e de Uso Sustentável e UCs Municipais de Proteção Integral e de Uso Sustentável.

O método estatístico de estimativa de densidades através do algoritmo Kernel foi aplicado para analisar a distribuição espacial, o padrão e a intensidade do fogo ao longo dos biomas, a partir da média da série temporal estudada. Todas as operações de geoprocessamento e elaboração de mapas foram realizadas utilizando o programa ArcGIS $10.3^{\circledR}$ do Programa de PósGraduação em Sensoriamento Remoto da Universidade Federal do Rio Grande do Sul, utilizando o Datum SIRGAS 2000.

Também foi analisada a tendência de crescimento (ou decrescimento) do número de focos de fogo, podendo ser indicada como natural ou não natural (causada pela atividade antrópica) aplicando-se o teste não paramétrico de Mann-Kendall (SAGARIKA; KALRA; AHMAD, 2014), que foi desenvolvido para detectar tendências em séries temporais cujos os dados são independentes e identicamente distribuídos, para um conjunto ordenado de $\mathrm{n}$ observações $\mathrm{X}=\mathrm{x}_{1}$, $\mathrm{x}_{2}, \ldots, \mathrm{x}_{\mathrm{n}}$, e dada conforme Sang, Wang e Liu (2014):

$$
\mathrm{S}=\sum_{\mathrm{i}<j} \operatorname{sgn}\left(\mathrm{x}_{\mathrm{j}}-\mathrm{x}_{\mathrm{i}}\right)
$$

Em que:

$$
\operatorname{sgn}\left(x_{j}-x_{i}\right)=\left\{\begin{array}{l}
-1, x_{j}>x_{i} \\
0, x_{j}=x_{i} \\
1, x_{j}<x_{i}
\end{array}\right.
$$

A hipótese nula $\mathrm{H}_{0}$ para o teste afirma que não há tendência na série temporal. Se $\mathrm{H}_{0}$ é verdadeira então $S$ segue uma distribuição normal com:

$$
E[S]=0
$$




$$
\operatorname{Var}[\mathrm{S}]=\frac{\mathrm{n}(\mathrm{n}-1)(2 \mathrm{n}+5)}{18}
$$

Em que: $E[S]$ é a média de $S$ e $V[S]$ é a variância de $S$. Assim, a estatística $Z$ de MannKendall é dada por:

$$
Z=\left\{\begin{array}{l}
\frac{S-1}{\sqrt{V[S]}} \text { para } S>0 \\
0 \text { para } S=0 \\
\frac{S+1}{\sqrt{V[S]}} \text { para } S<0
\end{array}\right.
$$

A estatística de teste $\mathrm{Z}$ dá o nível de significância para rejeitar a hipótese nula. A magnitude da tendência pode ser determinada através da aproximação de Theil-Sen (ATS). O coeficiente angular de ATS é dado por:

$$
\beta=\text { mediana }\left[\frac{x_{j}-x_{i}}{j-i}\right] \text { para todo } i \neq j
$$

A estatística S é fortemente ligada ao coeficiente $\tau$ de Kendall, dado por:

$$
\tau=\frac{S}{D}
$$

Em que D é dado por:

$$
D=\frac{n(n-1)}{2}
$$

Diz-se que a tendência é positiva se $\tau>0$ ou negativa se $\tau<0$.

Em seguida, foi aplicado o Teste de Mann-Kendall considerando Persistência de Longo Alcance (MK-PLA), uma vez que o Teste de Mann-Kendall exige a não persistência (correlação serial positiva) para série temporal analisada (HAMED, 2008). Portanto, os testes de tendência podem ser aplicados de duas formas: aplicando-se testes que considerem a correlação serial positiva ou removendo-se tal correlação e testando-se a tendência. Sendo assim, Hamed (2008) propôs uma modificação do Teste de Mann-Kendall que incorpora a persistência de longo alcance conforme indicado a seguir:

1. Cálculo do coeficiente de Hurst $(\mathrm{H})$.

a) Remoção da tendência da série utilizando-se coeficiente angular ATS.

b) Obtenção das variáveis normais $Z$ equivalentes dos ranks da série sem tendência através da equação: $Z_{i}=\phi^{-1}\left(\frac{R_{i}}{n+1}\right)$, em que Ri são os ranks da série sem tendência obtida anteriormente, n é o tamanho da série e $\phi^{-1}$ é a função transformada inversa da distribuição normal padrão.

c) A matriz de correlação para o dado coeficiente de Hurst é dado por $C_{n}(H)=\left[\rho_{|j-i|}\right]$ , para $\mathrm{i}=1: \mathrm{n}, \mathrm{j}=1: \mathrm{n} \mathrm{e}^{\rho_{l}=\frac{1}{2}\left(|l+1|^{2 H}-2|l|^{2 H}+|l-1|^{2 H}\right)}$ para $1>1$, em que $\rho_{l}$ é a função de autocorrelação do lag 1 para um dado $\mathrm{H}$. 
d) $\mathrm{O}$ valor de $\mathrm{H}$ é obtido maximizando numericamente a função de logverossimilhança de $\mathrm{H}: \log L(H)=-\frac{1}{2} \log \left|C_{n}(H)\right|-\frac{Z^{T}\left[C_{n}(H)\right]^{-1} Z}{2 \gamma_{0}}$, em que $\left|C_{n}(H)\right|$ é o determinante da matriz de correlação $\left[C_{n}(H)\right]^{-1}, Z^{T}$ é o vetor transposto de variáveis normais $Z$ equivalentes, $\left[C_{n}(H)\right]^{-1}$ é a matriz inversa e $\gamma_{0}$ é a variância dos $Z_{i}$.

2. Determinação do nível de significância de $\mathrm{H}$ a fim de se verificar se este é igual a 0,5 indicando uma série temporal aleatória, usando a média $\left(\mu_{H}\right)$ e o desvio padrão $\left(\sigma_{H}\right)$, em que $\mathrm{H}=0,5$ (distribuição normal) dado pelas equações equações $\mu_{H}=0,5-2,87 n^{-0,9067}$ e $\sigma_{H}=0,77654 n^{-0,5}-0,0062$.

3. Realização do cálculo da variância: se $\mathrm{H}$ é significante, a variância de $\mathrm{S}$ é calculada usando-se a equação $V(S)^{H}=\sum_{i<j} \sum_{k<l} \frac{2}{\pi} \sin ^{-1}\left(\frac{\rho|j-i|-\rho|i-l|-\rho|j-k|+\rho|i-k|}{\sqrt{(2-2 \rho|i-j|)(2-2 \rho|k-l|}}\right)$ Kendall é então calculada usando-se $V(S)^{H}$.

\section{Resultados e discussão}

Entre os biomas brasileiros, o Amazônico e o Cerrado foram os que apresentaram a maior quantidade de focos de fogo ao longo de toda a série temporal analisada, com valores ainda maiores de 2003 a 2007 chegando a atingir um valor de, respectivamente, 218.714 (em 2004) e 137.927 (em 2007) (Figura 2). Durante este período os demais biomas também tiveram um elevado registro, apresentando o maior número anual para a sequência de anos analisados. Já para a Caatinga, Mata atlântica e Pampa, a maior quantidade foi observada no ano de 2003, com $32.774,44.480$ e 1.800 focos, respectivamente, já no Pantanal, foi verificado no ano de 2005, o qual apresentou um número de 12.536. Contabilizando o total para os anos avaliados, constatou-se que a Amazônia obteve 1.846.098 locais com queima da vegetação, seguida do Cerrado (1.149.383), da Mata Atlântica (312.341) e Caatinga (291.194) que tiveram valores próximos. O Pantanal e Pampa apresentaram baixos valores quando comparados aos demais biomas com, respectivamente, 87.647 e 16.577 .

\section{Figura 2 - Quantidade de focos de fogo e área queimada $\left(\mathrm{km}^{2}\right)$ nos biomas brasileiros ao} longo da série temporal de 2003 a 2017

Figure 2 - Number of fire outbreaks and burned area $(\mathrm{km} 2)$ in Brazilian biomes over the time series from 2003 to 2017

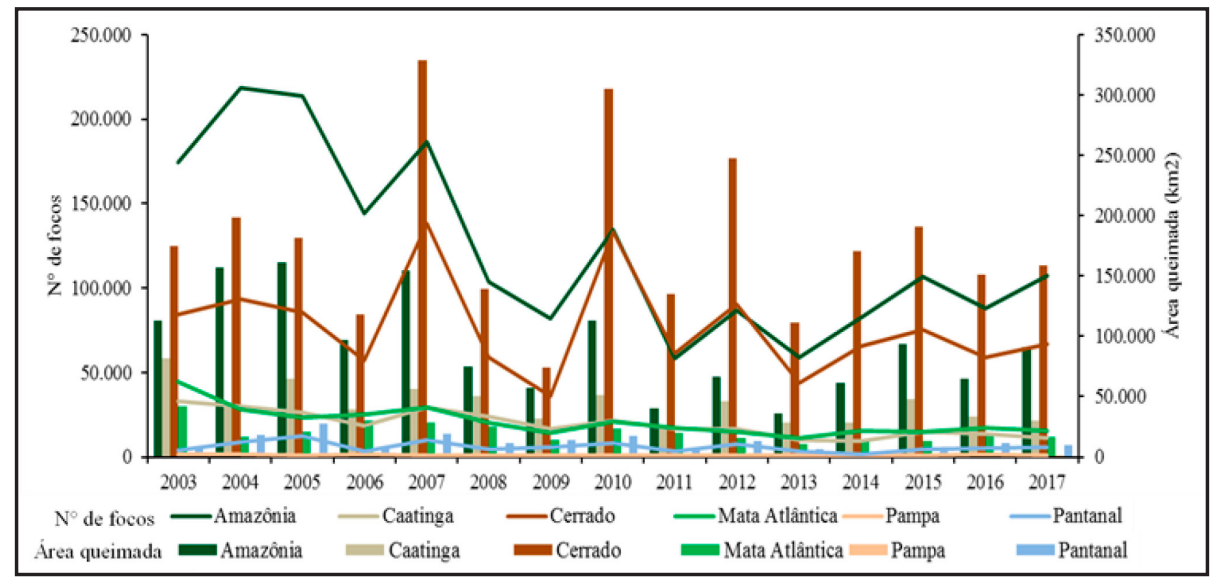

Fonte: Jesus et al. (2019) 
Nota-se uma oscilação anual em todos os biomas, havendo anos com acréscimos e outros com decréscimos ao longo da série temporal analisada, com alguns picos como pode-se constatar nos anos de 2010, 2012 e 2015. Comparando-se a quantidade de focos de fogo com o estudo realizado por Araújo, Ferreira e Arantes (2012), que utilizaram dados do sensor MODIS (Moderate Resolution Imaging Spectroradiometer) para analisar o padrão de distribuição de áreas queimadas nos biomas brasileiros de 2002 a 2010, nota-se grande semelhança no comportamento da distribuição das manchas de fogo ao longo da variação temporal analisada, com destaque aos picos no número de ocorrências evidenciados na Amazônia e Cerrado em 2004, 2005 e 2007, quando houve progressiva redução. Contudo, em 2010 o estudo dos autores não identificou um novo pico para o bioma Amazônico, registrando apenas o aumento na quantidade de queimadas para o Cerrado. Quanto aos demais biomas, observa-se uma similaridade na ocorrência das queimadas/incêndios. Já no estudo de Tasker e Arima (2016) também utilizando dados do MODIS foi observado um número muito elevado de eventos de incêndios no ano de 2007 (de uma série de 2001 a 2013) quando comparado aos presentes dados junto aos de Araújo, Ferreira e Arantes (2012).

Ao analisar a área queimada, observa-se que apesar da Amazônia ter sido o bioma com o maior registro de incidência de fogo, não foi o que teve maior área queimada para a série temporal analisada, ficando em segundo lugar com um total de $1.382 .264 \mathrm{~km}^{2}$, ficando atrás do Cerrado que apresentou uma área total de $2.685 .596 \mathrm{~km}^{2}$. Deve-se destacar que a Amazônia possui uma vegetação com menor deficit hídrico que o Cerrado, o que impede uma maior extensão de área queimada. Este comportamento quantitativo ao longo do tempo verificado se assemelha ao obtido por Araújo, Ferreira e Arantes (2012), com um maior destaque ao bioma Cerrado em comparação à Amazônia, e com pico de área queimada nos anos de 2007 e 2010 para ambos. Nestes mesmos anos, configurados como de seca, Tasker e Arima (2016) também constataram, na vegetação Amazônica, uma maior área com queima quando comparado aos demais períodos estudados.

Porém, o presente estudo se diferiu ao dos autores anteriormente citados principalmente para a Caatinga que, neste caso, identificou-se que apesar de menor número de focos de queimada/ incêndio que a Mata Atlântica, pode estar relacionada ao maior deficit hídrico na área de Caatinga se destacou sendo o terceiro bioma com a maior quantidade de área queimada $(655.845$ $\mathrm{km}^{2}$ ), seguido da Mata Atlântica com $307.860 \mathrm{~km}^{2}$, condição esta que pode estar relacionada ao maior deficit hídrico na área de Caatinga. O Pantanal e Pampa seguiram com as menores áreas queimadas, $180.346 \mathrm{~km}^{2}$ e $21.440 \mathrm{~km}^{2}$, respectivamente.

Anualmente, nota-se que as áreas queimadas estão relacionadas com o número de focos de fogo detectado, com maiores extensões de queima da vegetação nos anos de maior quantidade de incêndios. Além disso, foi observada uma tendência de redução da área queimada ao longo da série analisada. Entre os biomas, o Cerrado teve maior área queimada no ano de 2007 (329.138 $\left.\mathrm{km}^{2}\right)$, a Amazônia em $2004\left(157.007 \mathrm{~km}^{2}\right)$, a Caatinga, Mata Atlântica e Pampa em 2003 (82.048 $\mathrm{km}^{2}, 41.607 \mathrm{~km}^{2}, 2.488 \mathrm{~km}^{2}$, respectivamente), e o Pantanal em $2007\left(18.699 \mathrm{~km}^{2}\right)$.

Avaliando-se as a ocorrências de fogo mensalmente, constata-se que a variação sazonal da quantidade média tanto de foco como de área queimada são semelhantes para todos os biomas (Figura 3A e 3B). Apesar da Amazônia apresentar maior número de focos registrados, o Cerrado tem uma incidência mensal antecipada e com maior quantidade que este bioma, tendo 2 meses com valores superiores (maio e junho), com maior representatividade entre os meses de junho a outubro assim como constatado no estudo de Araújo e Ferreira (2015) e pico de queimadas em setembro como corroborado por Alvarado et al. (2017). Já na Amazônia os meses de maior ocorrência se dá entre os meses de agosto, setembro e outubro, conforme evidenciado também no estudo de Vasconcelos et al. (2013). 
Figura 3 - Média mensal do número de focos de fogo $(\mathrm{A})$ e de área queimada $\mathrm{em}_{\mathrm{km}}{ }^{2}(\mathrm{~B})$ por bioma de 2003 a 2017

Figure 3 - Monthly average of the number of fire outbreaks (A) and burned area in $\mathrm{km} 2$ (B) per biome from 2003 to 2017

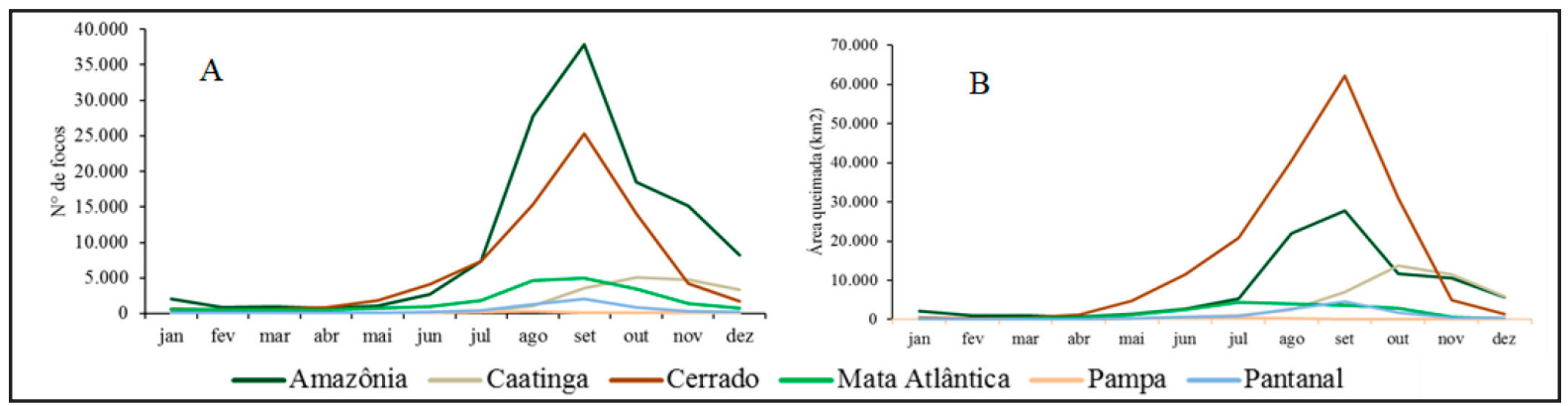

Fonte: Jesus et al. (2019)

De modo geral, há um acréscimo na quantidade de focos de fogo entre os meses de junho a dezembro, com o pico no mês de setembro para a maioria dos biomas: Cerrado (25.354), Amazônia (37.854), Mata Atlântica (4.939) e Pantanal (2.080), enquanto que o Pampa (272) que ocorre em agosto, e a Caatinga que é identificado no mês de outubro (5.126). Semelhante à distribuição do número de ocorrência de queima da vegetação ao longo dos meses, o comportamento da área queimada nos biomas também se dá em maior proporção de junho a dezembro. Também foi verificada uma relação entre o foco e a área queimada uma vez que os meses com os maiores registros de queimadas foram os mesmos com maiores áreas queimadas. Apesar disto, notou-se que o bioma Mata Atlântica e Pampa não tiveram essa relação, sendo observado, em ambos, uma maior extensão de fogo em julho.

Os biomas Amazônico e Cerrado foram os que apresentaram maior destaque em relação aos demais, e, portanto, foram os que tiveram maiores extensões referente à distribuição do fogo, conforme constatado também por Araújo, Ferreira e Arantes (2012), com forte atividade de incêndios ao longo da transição Amazônia-Cerrado, no território do estado do Maranhão e Pará, decorrente do uso intensivo da terra nesta área a qual é conhecida como Arco do Desmatamento. Observa-se que, espacialmente, a Amazônia tem baixa densidade de eventos de fogo na sua porção norte-oeste (apesar de já existir alguns locais com aumento da evidência de queimadas) enquanto o inverso é visto em toda a sua porção leste, com várias regiões consideradas com alta densidade (Figura 4). Tasker e Arima (2016) ao estudarem o regime de fogo na Amazônia de 2004 a 2013, também identificaram uma maior quantidade de focos e de área queimada também estão na porção leste e sul, ao longo da divisa com o Cerrado, e no leste do estado de Roraima, espacialmente semelhante ao verificado no presente estudo. Além disso, Bedia et al. (2015) observaram que as projeções meteorológicas de incêndios e suas anomalias futuras de 2026 a 2045 indicaram que, no Brasil, estas áreas se assemelham com as áreas de maiores manchas de densidade verificadas nas ocorrências do presente estudo (de 2003 a 2017), com destaque ao bioma Cerrado e Caatinga tendo maiores magnitudes e intensidades das anomalias. 


\section{Figura 4 - Densidade média da ocorrência de fogo ao longo dos biomas brasileiros e nas Unidades de Conservação por meio do método Kernel de 2003 a 2017}

Figure 4 - Average density of fires occurrence along the Brazilian biomes and in Conservation Units using the Kernel method from 2003 to 2017

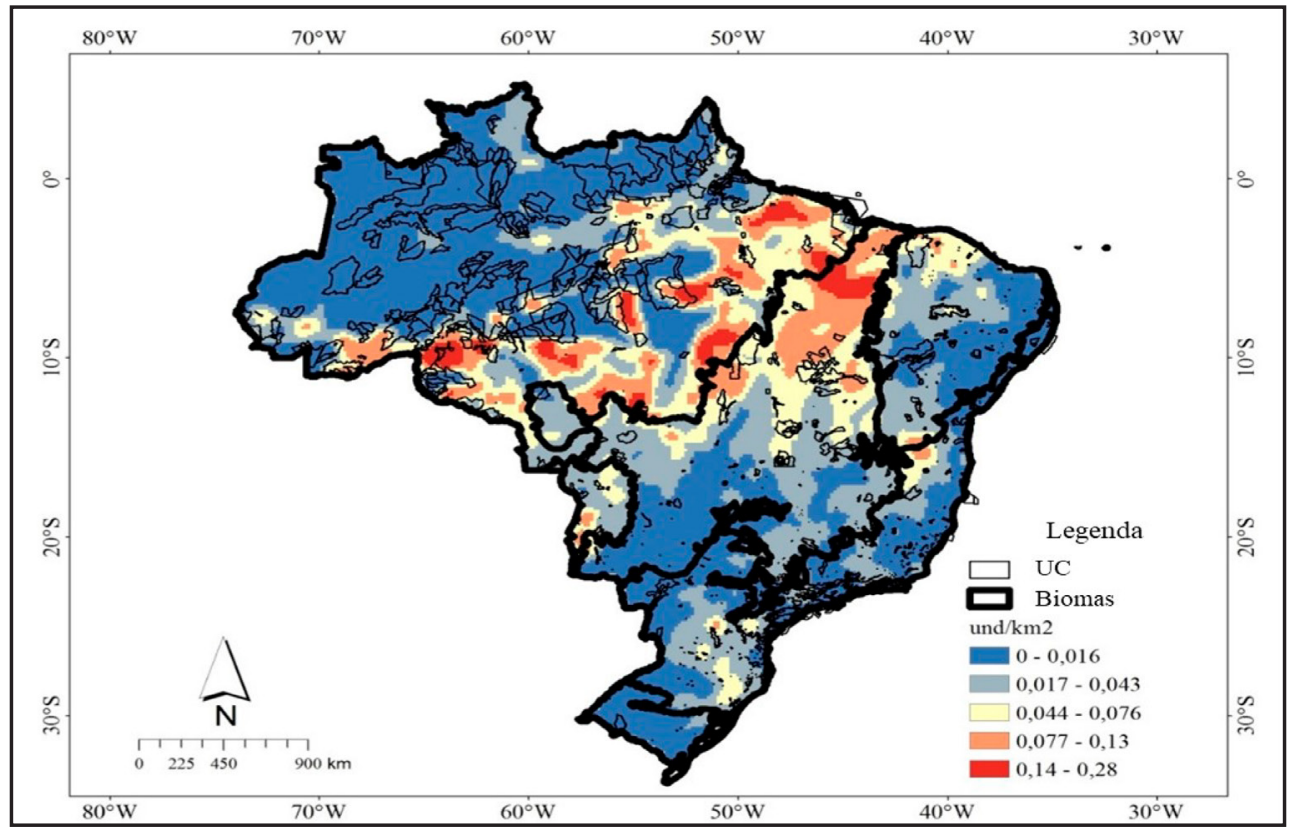

Fonte: Jesus et al. (2019)

No Cerrado, constatou-se uma região com maior densidade da incidência de fogo, situada ao norte do bioma, fazendo divisa com a Amazônia e Caatinga, com destaque ao estado de Tocantins que teve grande parte de sua área com queimadas ou incêndios florestais, e que, segundo Carvalho et al. (2017), tem as áreas compreendidas na Ilha do Bananal e o Jalapão com as maiores ocorrências e áreas queimadas, locais estes associados aos maiores Produto Interno Bruto agropecuário do Estado. Além disto, toda a porção norte foi a que apresentou os mais altos níveis de ocorrência do fogo. Na Caatinga, nota-se que a região leste apresentou uma baixa densidade, contudo, a porção noroeste e sul tiveram regiões de alta densidade, que apesar de não apresentar extensas áreas com a mais alta densidade, comparado aos biomas anteriores, tiveram grande dimensão no território com densidade que indicam uma região de alerta para a quantidade de registro de focos detectados. Na Mata Atlântica, também foi observada a mesma condição de distribuição e extensão relacionado à alta densidade da Caatinga, verificando-se maiores densidades de queimadas na divisa com o sul da Caatinga e na região mais ao sul do bioma. No Pantanal, observaram-se duas regiões com forte atuação de fogo (norte e sul do bioma), enquanto as demais áreas de ocupação da sua vegetação apresentaram baixa incidência, porém, também com destaque para possíveis aumentos na sua quantificação. No Pampa, constatou-se uma baixa densidade de ocorrência, com uma porção ao norte do bioma sendo identificado fora desse nível, entretanto, sem elevada densidade.

Assim como no número de focos de queimada/incêndio, a Amazônia foi o bioma que apresentou a maior quantidade nas Unidades de Conservação com um total de 159.931 registros, seguido do Cerrado com 95.673 e Caatinga com 25.864, valor este superior à Mata Atlântica que teve um total de 17.821 (Tabela 1). O Pantanal e Pampa, por sua vez, tiveram os menores números, com 2.143 e 121 , respectivamente. Deve-se considerar também para esse levantamento que existe uma variação tanto no número como no tamanho das Unidades de Conservação para cada bioma, refletindo também no registro de ocorrência da atuação do fogo. Entre os biomas estudados, a Mata Atlântica tem a maior 
quantidade de áreas protegidas (879), contudo, está na quarta posição entre as que tiveram os maiores pontos com fogo. A Amazônia se destacou tendo o maior registro de fogo com 159.931 nas 286 UCs. Os elevados números de queimadas nestas áreas chamam a atenção, o que é ainda mais preocupante tendo em vista a baixa eficiência quanto ao combate aos incêndios florestais nas UCs do Brasil (LIMA et al., 2018).

\section{Tabela 1 - Focos de fogo detectados nas Unidades de Conservação de âmbito Federal, Estadual e Municipal para cada bioma de 2003 a 2017}

Table 1 - Fire outbreaks detected in the Conservation Units of Federal, State and Municipal scope for each biome from 2003 to 2017

\begin{tabular}{lccccccc}
\hline \multicolumn{7}{c}{ Unidades de Conservação } \\
\hline \multicolumn{1}{c}{ Federal } & Estadual & \multicolumn{2}{c}{ Municipal } \\
\hline \multicolumn{1}{c}{ Bioma } & PI & US & PI & US & PI & US & Total \\
\hline Amazônia & $18.464(41)$ & $47.584(98)$ & $4343(35)$ & $89.056(98)$ & $8(4)$ & $476(10)$ & $159.931(286)$ \\
Caatinga & $1.590(17)$ & $18.510(58)$ & $491(28)$ & $5.273(34)$ & $0(1)$ & $0(1)$ & $25.864(139)$ \\
Cerrado & $32.512(22)$ & $11.497(71)$ & $5.470(80)$ & $45.940(81)$ & $32(12)$ & $222(8)$ & $95.673(274)$ \\
Mata Atlântica & $1.839(53)$ & $3.702(204)$ & $1.360(169)$ & $10.409(273)$ & $87(112)$ & $424(68)$ & $17.821(879)$ \\
Pampa & $40(2)$ & $26(3)$ & $15(6)$ & $40(1)$ & $0(4)$ & $0(1)$ & $121(17)$ \\
Pantanal & $324(2)$ & $24(2)$ & $1450(4)$ & $338(5)$ & $7(1)$ & $-(-)$ & $2143(14)$ \\
\hline Total & $54.769(137)$ & $81.343(436)$ & $13.129(322)$ & $151.056(492)$ & $134(134)$ & $1.122(88)$ & $301.553(1.609)$ \\
\hline
\end{tabular}

Fonte: Autores (2019)

Em que: PI = Proteção Integral, e US = Uso Sustentável. Valores entre parênteses representam o total de Unidades de Conservação avaliadas nos diferentes entes federativos para cada bioma.

Em termos de total de queimadas/incêndios nos diferentes tipos das 1.609 Unidades de Conservação analisadas, observa-se um maior número do tipo de Uso Sustentável (US) que por sua vez obtiveram uma maior quantidade de focos quando comparado às de Proteção Integral (PI) nos três âmbitos federativos, diferentemente do verificado por Torres et al. (2016) ao analisarem o perfil dos incêndios florestais de 2008 a 2012 nas Unidades de Conservação do Brasil que indicaram um maior impacto do fogo nas UCs de Proteção Integral, com destaque à região Norte do país que apresentou maior área queimada, apesar de menor número de ocorrências, e também aos estados do Ceará e Tocantins onde foram contabilizados maior incidência e área queimada, respectivamente, nas suas áreas protegidas. Comparando-se os tipos de áreas protegidas nos diferentes níveis federativos, constata-se uma maior ocorrência nas de US em relação aos biomas, com exceção das UCs Federais no Cerrado, Pampa e Pantanal, este também teve maior registro de fogo nas de PI. Além disso, foi verificado que entre as de Proteção Integral, apesar de maior número de UCs de nível Estadual (322) comparada com as de responsabilidade Federal (137), estas tiveram mais registros (54.769).

Entre os biomas, nota-se, ao longo da série temporal estudada, que as Unidades de Conservação na Amazônia que mais apresentaram focos de fogo foram as Áreas de Proteção Ambiental (APA) Triunfo do Xingu (36.444), do Arquipélago do Marajó (11.956) e da Baixada Maranhense (11.209), todas Estaduais de Uso Sustentável. Já as de Proteção Integral foram: a Estação Ecológica da Terra do Meio (2.783), a Reserva Biológica do Gurupi (2.352) e a Reserva Biológica Nascentes Serra do Cachimbo (2.200). Já no Cerrado, foram as Áreas de Proteção Ambiental Estaduais do Rio Preto (11.007) e Ilha do Bananal/Cantão (10.881), ambas de Uso Sustentável, junto com as Federais de Proteção Integral: os Parques Nacional das 
Nascentes do Rio Parnaíba (10.545) e do Araguaia (6.704), e a Estação Ecológica Serra Geral do Tocantins (5.348). As Áreas de Proteção Ambiental de Uso Sustentável da Serra da Ibiapaba (10.216), Chapada do Araripe (5.904), Dunas e Veredas do Baixo São Francisco (2.212) e Delta do Parnaíba (1.647) tiveram os maiores registros de fogo no bioma Caatinga. Na Mata Atlântica foi identificada uma alta quantidade de focos principalmente nas UC Estaduais de Uso Sustentável, como as Áreas de Proteção Ambiental do Alto do Mucuri (1.234), da Serra da Esperança (873) e do Sistema Cantareira (813), porém, a de cunho Federal Ilhas e Várzeas do Rio Paraná 1.846 foi a UC com maior número registrado. No Pampa, a Área de Proteção Ambiental do Banhado Grande (40), Estação Ecológica do Taim (38) e a APA Ibirapuitã (26) foram as que mais apresentaram focos de fogo. Já no bioma Pantanal, estes focos foram detectados em maior número nas UCs de Proteção Integral, encontrados nos Parques Estaduais Encontro das Águas (583), do Guirá (498) e do Pantanal do Rio Negro (367), conjuntamente com o Parque Nacional do Pantanal Mato-grossense (298). Apesar de diferente proporção entre as UC com maior incidência de atuação do fogo quando comparado ao levantamento realizado por Torres et al. (2016), constatou-se que, semelhantemente, algumas áreas protegidas se destacaram como: a Estação Ecológica Serra Geral do Tocantins (Tocantins), Estação Ecológica do Taim (Rio Grande do Sul) e os Parques Nacional das Nascentes do Rio Parnaíba (Piauî) e do Araguaia (Tocantins).

Analisando a tendência estatística da ocorrência das queimadas/incêndios nos biomas do Brasil, observa-se no Teste de Mann-Kendall (Tabela 2) que o Pampa apresentou o maior valor $(0,158)$ e foi o único com p inferior a 0,05 , indicando evidência de tendência positiva, foi a única formação vegetal que foi necessário refazer a análise utilizando a persistência de longo alcance, enquanto que para os outros biomas inexiste uma tendência temporal. Os demais biomas apresentaram baixos valores para o Teste em questão, e mesmo o Cerrado tendo o segundo maior valor $(0,064)$ não apresentou evidência estatística para configurar uma tendência não natural. Caúla et al. (2017), também utilizando este teste verificaram valores significativos de focos de fogo no estado do Rio de Janeiro para o ano de 2001 de série de 2000 a 2013, com tendência de aumento para os anos de 2001 e 2013. Pinto (2014), analisando globalmente as tendências em contagens de fogos noturnos ativos (1996-2012) observou que, de modo geral, não foi detectada nenhuma tendência significativa, identificando apenas pequenas áreas com ligeiras tendências. Da mesma forma, Arino, Casadio e Serpe (2012) também constataram a inexistência de tendência na quantidade de incêndios em nível global, indicando que só são verificadas tendências positivas e negativas em análises em menores escalas.

Tabela 2 - Valores da análise estatística de tendência aplicada na série de focos de queimadas nos biomas estudados

Table 2 - Trend statistical analysis values applied in the series of burnout outbreaks in the studied biomes

\begin{tabular}{|c|c|c|c|c|c|}
\hline & \multicolumn{2}{|c|}{ MK } & \multicolumn{2}{|c|}{ Hurst } & \multirow{2}{*}{$\frac{\text { MK-PLA }}{\mathrm{P}}$} \\
\hline & Tau & $\mathrm{P}$ & $\mathrm{H}$ & $\mathrm{P}$ & \\
\hline Amazônia & 0,008 & 0,853 & 0,995 & $<0,001$ & 0,235 \\
\hline Caatinga & 0,042 & 0,332 & 0,996 & $<0,001$ & 0,556 \\
\hline Cerrado & 0,064 & 0,147 & 0,996 & $<0,001$ & 0,546 \\
\hline Mata Atlântica & 0,031 & 0,482 & 0,994 & $<0,001$ & 0,386 \\
\hline Pantanal & 0,012 & 0,785 & 0,993 & $<0,001$ & 0,413 \\
\hline Pampa & 0,158 & $<0,001$ & 0,935 & $<0,001$ & 0,230 \\
\hline
\end{tabular}

Fonte: Autores (2019)

Em que: $\mathrm{MK}=$ Teste de Mann-Kendall; $\mathrm{H}=$ Expoente de Hurst; MK-PLA = Teste de Mann-Kendall com Persistência de Longo Alcance. 
Os dados observados no Expoente Hurst indicaram que existe memória na série do fenômeno avaliado, ou seja, que há repetição do padrão ao longo do tempo, indicando a persistência ou recorrência da sequência das queimadas para todos os biomas, uma vez que todos os valores foram superiores a 0,5 e que séries com $0,5>\mathrm{H} \geq 1$ indicam que possuem memória de longo alcance. Já no Teste de Mann-Kendall com Persistência de Longo Alcance constata-se que há uma variabilidade natural do processo, e, portanto, a tendência ao longo do tempo apresenta evidências de associação às causas naturais, podendo-se inferir que é resultado das condições climáticas (mesmo não sabendo quais interferem em magnitude), não havendo indicação estatística de tendência de crescimento de focos por origem antrópica uma vez que em todos os biomas o valor de $\mathrm{p}$ foi superior a 0,05 .

Ao analisar o efeito da sazonalidade climática do fogo nos biomas brasileiros, Araújo, Ferreira e Arantes (2012) também verificaram a influência de fenômenos meteorológicos, como dados de precipitação junto a fenômenos como El Niño e La Niña, e identificaram relação entre estes e a distribuição do fogo nessas formações vegetais. Especificamente na região Amazônica, Tasker e Arima (2016) também indicam que há evidências diretas da atividade pluviométrica na ocorrência do fogo. Aragão et al. (2007) verificaram que no caso do aumento da frequência de secas na região Amazônica, a ocorrência da dispersão do fogo pode ser o principal fator de modificação nesta floresta. E como estes autores identificaram que as regiões norte, central e leste da Amazônia estão associadas aos eventos de oscilações do El Niño. Da mesma forma, Cardozo et al. (2014) observaram que, em geral, os anos de menores volumes de precipitação foram os que tiveram maiores áreas queimadas ao analisarem a distribuição espacial e temporal da área queimada na floresta Amazônica no Estado de Rondônia utilizando dados do sensor MODIS durante os anos de 2000 a 2011.

Araújo e Ferreira (2015) também notaram maiores áreas queimadas no Cerrado no período de seca, principalmente na metade desta estação (ALVES; PÉREZ-CABELLO, 2017). Esta relação se dá, pois, as condições meteorológicas predominantes em ecossistemas ou momentos mais áridos geralmente são mais favoráveis à propagação do fogo, apesar de terem menor material combustível, enquanto o inverso é limitado pelas condições de umidade (BEDIA et al., 2015).

Sendo assim, outro fator a ser considerado no estudo do padrão do fogo é a característica das diferentes tipologias vegetais, uma vez que o padrão da distribuição da sua queima está associado ao material combustível como a sua carga e estrutura, existindo vegetações mais susceptíveis ao fogo (MAGALHÃES et al., 2017). Além disto, deve-se considerar a influência das atividades humanas nesses eventos, como observado por Araújo, Ferreira e Arantes (2012), uma vez que até mesmo o fogo ocasionado pela condição climática pode ser intensificado pelo uso da terra (CARDOZO et al., 2014), pois a atuação antrópica faz-se o uso do fogo na queima de florestas ou manejo de culturas agrícolas ou pastagens (VASCONCELOS et al., 2013). Além da relação do tipo e condições da vegetação na dispersão do fogo, outras características também podem influenciar neste processo, como: topografia, histórico do evento e o próprio clima precisam ser levados em conta para definir estratégias eficazes de manejo, principalmente em Unidades de Conservação (FRANKE et al., 2018).

Portanto, é necessária a aplicação de uma Política Nacional clara de gestão de combate a incêndios e estratégias visando à conservação, como por exemplo: a inclusão do fogo nos planos de manejo das áreas protegidas, treinamento para o planejamento e a aplicação de fogo na vegetação, fomento governamental para pesquisas e experimentação em incêndios, e até mesmo com a divulgação dos benefícios do fogo para a conservação ambiental como no caso do Cerrado (DURIGAN; RATTER, 2016). 


\section{Conclusões}

O estudo permitiu identificar as regiões com maiores focos de fogo em cada Bioma brasileiro, com destaque ao Cerrado e Amazônia, observando espacialmente o seu padrão de distribuição e indicando a maior densidade de incidência na transição Amazônia-Cerrado e divisa com a Caatinga, alertando também a esta fitofisionomia quanto à ocorrência deste evento. Além disso, foi possível quantificar o número em cada Unidade de Conservação de Proteção Integral (PI) e de Uso Sustentável (US) no âmbito: Federal, Estadual e Municipal em cada respectivo bioma para a série temporal estudada, sobressaltando as de Uso Sustentável devido a uma elevada ocorrência, sendo as mais vulneráveis em todas as esferas institucionais, destacando-se as Áreas de Proteção Ambiental (APA) Estaduais Triunfo do Xingu, do Arquipélago do Marajó e Baixada Maranhense, todas na Amazônia, como sendo as que tiveram maior registro de fogo.

A avaliação da tendência estatística evidenciou a sazonalidade da ocorrência do fogo em todos os biomas estudados, o que indica a influência das condições climáticas no padrão temporal, bem como serem as épocas de maiores atividades do homem sobre as áreas vegetadas. Notou-se ainda que não houve crescimento dos focos das queimadas/incêndios em todos os Biomas, exceto o Pampa, cuja causa do crescimento detectada foi natural, contudo, não se pode descartar ser intensificada pela atuação antrópica em menor magnitude.

Portanto, são importantes e necessárias medidas que visem minimizar as atividades humanas que facilitem o surgimento das queimadas irregulares, e o monitoramento contínuo de incêndios florestais em cada tipo de vegetação do país, a fim de reduzir os impactos causados nos diferentes ecossistemas, criando políticas públicas de prevenção e melhor atuação de controle do fogo, garantindo segurança à população que pode, por ventura, ter problemas ocasionados pela sua expansão descontrolada, necessitando também de um maior foco de proteção e intervenção em relação à sua propagação nas Unidades de Conservação.

\section{Referências}

ALVARADO, S. T. et al. Drivers of fire occurrence in a mountainous Brazilian cerrado savanna: Tracking long-term fire regimes using remote sensing. Ecological Indicators, Amsterdam, v. 78, p. 270-281, jul. 2017.

ALVES, D. B.; PÉREZ-CABELLO, F. Multiple remote sensing data sources to assess spatio temporal patterns of fire incidence over Campos Amazônicos Savanna Vegetation Enclave (Brazilian Amazon). Science of the Total Environment, Amsterdam, v. 601-602, p. 142-158, mai. 2017.

ARAGÃO, L. E. O. C. et al. Spatial patterns and fire response of recent Amazonian droughts. Geophysical Research Letters, Medford, v. 34, p. 1-5, abr. 2007.

ARAÚJO, F. M.; FERREIRA, L. G.; ARANTES, A. A. Distribution patterns of burned areas in the brazilian biomes: an analysis based on satellite data for the 2002-2010 period. Remote Sensing, Basel, v. 4, p. 1929-1946, jun. 2012.

ARAÚJO, F. M.; FERREIRA, L. G. Satellite-based automated burned área detection: A performance assessment of the MODIS MCD45A1 in the Brazilian savanna. International 
Journal of Applied Earth Observation and Geoinformation, Amsterdam, v. 36, p. 94-102, abr. 2015.

ARINO, O.; CASADIO, S.; SERPE, D. Global night-time fire season timing and fire count trends using the ATSR instrument series. Remote Sensing of Environment, Amsterdam, v. 116, p. 226238 , jan. 2012.

BEDIA, J. et al. Global patterns in the sensitivity of burned area to fire-weather: Implications for climate change. Agricultural and Forest Meteorology, Amsterdam, v. 214-215, p. 369-379, set. 2015.

BRASIL. MINISTÉRIO DA AGRICULTURA. Levantamento exploratório/reconhecimento de solos do Estado da Paraíba. Rio de Janeiro: SUDENE/DRN, Convênio MA/CONTAP/USAID/ Brasil, 1972. $670 \mathrm{p}$.

CARDOZO, F. S. et al. Analysis and Assessment of the Spatial and Temporal Distribution of Burned Areas in the Amazon Forest. Remote Sensing, Basel, v. 6, p. 8002-8025, ago. 2014.

CARVALHO, E. V. et al. Caracterização de áreas queimadas no estado do Tocantins no ano de 2014. Revista Floresta, Curitiba, v. 47, n. 3, p. 269-278, jul.-set. 2017.

CAÚLA, R. H. et al. Nonparametric statistics applied to fire foci obtained by meteorological satellites and their relationship to the MCD12Q1 product in the state of Rio de Janeiro, Southeast Brazil. Land Degradation \& Development, Medford, v. 28, p. 1056-1067, jun. 2017.

COSTA, E. P. et al. Incêndios florestais no entorno de unidades de conservação - estudo de caso na Estação Ecológica de Águas emendadas, Distrito Federal. Ciência Florestal, Santa Maria, v. 19, n. 2, p. 195-206, abr.-jun. 2009.

DURIGAN, G.; RATTER, J. A. The need for a consistent fire policy for Cerrado conservation. Journal of Applied Ecology, Londres, v. 53, p. 11-15, out. 2016.

EMBRAPA. Agência de Informação Embrapa Bioma Cerrado. Tipos de Vegetação do Bioma Cerrado. Disponível em: http://www.agencia.cnptia.embrapa.br/Agencia16/AG01/arvore/ AG01_23_911200585232.html. Acesso em: 19 ago. 2018.

FRANKE, J. et al. Fuel load mapping in the Brazilian Cerrado in support of integrated fire management. Remote Sensing of Environment, Amsterdam, v. 217, p. 221-232, ago. 2018.

HAMED, K. H. Trend detection in hydrologic data: The Mann-Kendall trend test under the scaling hypothesis. Journal of Hydrology, Amsterdam, v. 349, p. 350-363, fev. 2008.

IBGE. Instituto Brasileiro de Geografia e Estatística. Mapa de Biomas e de Vegetação. 2004. Disponível em: http://www.ibge.gov.br/home/presidencia/noticias/21052004biomashtml.shtm. Acesso em: 19 ago. 2018.

INPE. Instituto Nacional de Pesquisas Espaciais. Programa Queimadas. Disponível em: http:// www.inpe.br/queimadas/portal. Acesso em: 14 ago. 2018.

LIMA, G. S. et al. Avaliação da eficiência de combate aos incêndios florestais em Unidades de Conservação brasileiras. Revista Floresta, Curitiba, v. 48, n. 1, p. 113-122, jan.-mar. 2018.

MAGALHÃES, S. R. et al. Comportamento do fogo em diferentes períodos e configurações de uma paisagem no nordeste de Portugal. Ciência Florestal, Santa Maria, v. 27, n. 2, p. 457-469, abr.-jun. 2017.

MMA. Ministério do Meio Ambiente. Sistema Nacional de Unidades de Conservação da Natureza. Lei no 9.985, de 18 de julho de 2000. Decreto n⿳⺈ 4.340, de 22 de agosto de 2002. Decreto no 5.746, de 5 de abril de 2006. Disponível em: http://www.mma.gov.br/images/arquivos/areas_ protegidas/snuc/Livro\%20SNUC\%20PNAP.pdf. Acesso em: 26 ago. 2018. 
MMA. Ministério do Meio Ambiente. Biomas. Disponível em: http://www.mma.gov.br/biomas. Acesso em: 19 ago. 2018a.

MMA. Ministério do Meio Ambiente. Download de dados geográficos. Disponível em: http:// mapas.mma.gov.br/i3geo/datadownload.htm. Acesso em: 15 ago. 2018b.

PINTO, D. M. S. S. Análise global de tendências em contagens de fogos noturnos ativos (1996 - 2012). 2014. 59 f. Dissertação (Mestrado em Engenharia Florestal e dos Recursos Naturais) Universidade de Lisboa, Lisboa. 2014.

SAGARIKA, S.; KALRA, A.; AHMAD, S. Evaluating the effect of persistence on long-term trends and analyzing step changes in streamflows of the continental United States. Journal of Hydrology, Amsterdam, v. 517, p. 36-53, set. 2014.

SANG, Y.-F.; WANG, Z.; LIU, C. Comparison of the MK test and EMD method for trend identification in hydrological time series. Journal of Hydrology, Amsterdam, v. 510, p. 293-298, mar. 2014.

SANTOS, J. F. C. et al. Potentials and limitations of remote fire monitoring in protected areas. Science of the Total Environment, Amsterdam, v. 616-617, p. 1347-1355, mar. 2018.

TASKER, K. A.; ARIMA, E. Y. Fire regimes in Amazonia: The relative roles of policy and precipitation. Anthropocene, Amsterdam, v. 14, p. 46-57, abr. 2016.

TORRES, F. T. P. et al. Perfil dos incêndios florestais em Unidades de conservação Brasileiras no período de 2008 a 2012. Revista Floresta, Curitiba, v. 46, n. 4, p. 531-542, out.-dez. 2016.

VASCONCELOS, S. S. et al. Variability of vegetation fires with rain and deforestation in Brazil's state of Amazonas. Remote Sensing of Environment, Amsterdam, v. 136, p. 199-209, set. 2013. 